ఠ

\title{
Novel targeted therapies for resistant ALK- rearranged non-small-cell lung cancer: ceritinib and beyond
}

This article was published in the following Dove Press journal:

OncoTargets and Therapy

20 April 2015

Number of times this article has been viewed

\author{
Zeyad Kanaan' \\ Goetz H Kloecker' \\ Ajit Paintal $^{2}$ \\ Cesar A Perez' \\ 'Division of Medical Oncology \\ and Hematology, James Graham \\ Brown Cancer Center, University \\ of Louisville, Louisville, KY, \\ ${ }^{2}$ Department of Pathology, \\ Northwestern University, Feinberg \\ School of Medicine, Chicago, IL, USA
}

Correspondence: Cesar A Perez Division of Medical Oncology and Hematology, James Graham Brown Cancer Center, University of Louisville, 529 South Jackson Street, Suite 426, Louisville, KY 40202, USA

Tel +l 5025624369

Fax + I 502562 68II

Email capere06@louisville.edu

\begin{abstract}
Lung cancer is the leading cause of cancer-related mortality in both sexes, accounting for over one quarter of cancer deaths. Non-small-cell lung cancer (NSCLC) comprises $85 \%-90 \%$ of lung cancer diagnoses and despite advances in multimodality therapies, 5-year survival rates remain dismal with a median survival for patients with metastatic disease of 1 year. The positive outcomes of targeted therapies against the kinase domain of epidermal growth factor receptor in NSCLC triggered consistent efforts to identify the so-called driver mutations as other potential targets. Anaplastic largecell kinase (ALK) gene rearrangements were identified and targeted resulting in promising response rates in early studies. Unfortunately, most of the patients treated with crizotinib, the first-generation ALK inhibitor, progressed within 9 months. Ceritinib is a second-generation ALK inhibitor that has demonstrated activity in crizotinib-resistant patients, becoming a promising treatment option in this population. Furthermore, additional novel ALK inhibitors and agents targeting alternative pathways have been recruited to rechallenge this evasive disease post-crizotinib resistance.
\end{abstract}

Keywords: crizotinib, EML4-ALK rearrangement, Vysis, ALK inhibitor

\section{EML4-ALK gene fusion in non-small-cell lung cancer}

Anaplastic large-cell kinase (ALK) protein is a transmembrane "dependence receptor" that is pro-apoptotic in its inactive, ligand-free state and anti-apoptotic in its active, ligand-bound state. It is composed of a large extracellular domain and an intracellular kinase domain., ${ }^{1,2}$ Inversions in chromosome $2 \mathrm{p}$ can lead to fusion genes with the kinase domain of ALK, turning the transmembrane ligand-activated receptor into an intracellular fusion protein with autonomous mitogenic signaling. The fusion protein formed from the $\mathrm{N}$-terminus of EML4, a protein which belongs to the family of echinoderm microtubule-associated protein-like proteins, and the intracellular kinase-containing domain of ALK was the first fusion protein detected by Soda et al in 2007 in a resected adenocarcinoma specimen obtained from a 62-year-old male smoker. ${ }^{3}$ The EML4-ALK fusion protein is the most common fusion protein and is found in approximately $5 \%$ of lung adenocarcinomas. ${ }^{49}$ Its occurrence was more commonly detected in younger patients, non- or light smokers, tumors with solid histology, and late-stage disease, and considered almost always mutually exclusive of KRAS and epidermal growth factor receptor (EGFR) mutations. ${ }^{10}$ Non-EML4ALK fusion partners also exist, including reported partners as KIF5B and TGF. ${ }^{11,12}$

\section{Testing for ALK rearrangements in clinical practice}

At the current time, the only US Food and Drug Administration (FDA)-approved companion diagnostic test for the detection of ALK rearrangements in metastatic 
non-small-cell lung cancer (NSCLC) is the Vysis ALK fluorescent in situ hybridization (FISH) probe kit (Abbott Molecular, Des Plaines, IL, USA). ${ }^{13}$ As this is a break-apart FISH assay, the test is capable of detecting ALK rearrangements irrespective of the fusion partner.

While some institutions have employed and described ALK FISH testing in alcohol-fixed and air-dried cytology specimens, the Vysis ALK FISH probe kit is only validated for use on formalin-fixed paraffin-embedded tissue (FFPE). As such, generating FFPE which can be used for all the FDA-approved EGFR and ALK companion diagnostic testing should be a priority at the time of specimen triage. All histologic specimens are routinely processed as FFPE, and most cytology specimens, particularly effusion specimens and aspirates, can be processed as FFPE.

Alternative modalities for ALK testing include immunohistochemistry (IHC) and reverse transcription-polymerase chain reaction (RT-PCR)-based assays. IHC using the Dako ALK antibody that is employed at most institutions is not sensitive enough to detect the low levels of ALK expression found in most cases of ALK-rearranged NSCLC. Newer antibodies (D5F3 and 5A4) from Ventana (Roche, Switzerland), Novacastra (Leica Biosystems, Germany), and Cell Signaling (Danvers, MA, USA) are extremely sensitive and have been found to have excellent specificity. ${ }^{14}$

\section{Crizotinib activity in NSCLC- harboring EML4/ALK translocations}

Crizotinib, initially developed as a c-Met inhibitor, is an oral small molecule inhibitor of ALK tyrosine kinase which showed significant overall response rates (ORRs) in early clinical trials. ${ }^{15}$ In 2006, prior to the discovery of the EML4ALK rearrangement in NSCLC, the Phase I single-arm multicenter PROFILE 1001 trial was developed, initially enrolling patients with advanced solid tumors refractory to standard treatments to study the adverse-effect (AE) profile and maximal tolerated dose of crizotinib. An unexpected observation of significant tumor response in two patients with NSCLC who harbored the ALK gene rearrangement in the dose-escalation period prompted the enrollment of an expanded molecular cohort of 82 out of 1,500 patients with advanced NSCLC prospectively identified to harbor the ALK gene rearrangement. In this cohort, an ORR of $57 \%$ was observed, and $33 \%$ of patients had stable disease. The mean duration of treatment was 6.4 months at the time of data cut-off in February 2010. At the time, 77\% of patients were still receiving treatment. ${ }^{15}$ An expansion of this trial recruited additional patients to this cohort until June 1, 2011 for a total of 143 patients with evaluable disease. The ORR was $60.8 \%$ (87 patients) including three patients with a complete response (CR) and 84 with a partial response (PR). Objective responses were detected as early as 2 weeks from the start of treatment with a median of 7.9 weeks. The median duration of response was 49.1 weeks and was independent of age and sex. Responses were seen with a higher proportion of patients with poor Eastern Cooperative Oncology Group performance status scores, previous multiple lines of treatment, and Asians. The estimated median progression-free survival (PFS) was 9.7 months for all patients and 18.3 months for patients treated with first-line crizotinib $(n=24)$. Median overall survival (OS) rates had not been reached by the time of data cut-off, yet the preliminary estimated 6-month and 12-month OS rates were $87.9 \%$ and $74.8 \%$, respectively. The majority of the cohort experienced AEs of treatment, mainly grade 1 or 2 . The most frequently occurring AEs were visual effects, gastrointestinal effects, and peripheral edema. Gastrointestinal and visual AEs generally decreased over time, whereas peripheral edema increased with continuing treatment. ${ }^{16}$ With these results, crizotinib received conditional accelerated approval for the treatment of ALK-rearranged NSCLC in October 2011.

On November 20, 2013, the FDA granted regular approval for crizotinib in the treatment of metastatic ALK-rearranged NSCLC after progression on first-line standard of care chemotherapy based on the results of the Phase III PROFILE 1007 study demonstrating superior PFS and ORR compared to standard chemotherapy. In this prospective randomized trial, 347 patients with locally advanced or metastatic ALK-rearranged NSCLC who progressed on a platinum-based regimen were randomized to receive either oral crizotinib $250 \mathrm{mg}$ twice daily $(n=173)$ or intravenous chemotherapy with pemetrexed or docetaxel $(n=174)$. Cross-over to crizotinib was allowed in patients who progressed on the chemotherapy arm and were then included in the ongoing Phase II PROFILE 1005 study. The results of the PROFILE 1007 study revealed a 4.7-month PFS benefit (7.7 months vs 3.0 months; hazard ratio [HR] $0.49,95 \%$ confidence interval [CI] 0.37-0.64, $P<0.0001$ ) and a $45 \%$ superior ORR $(65 \%$ vs $20 \%, P<0.0001)$ with crizotinib compared to chemotherapy. ${ }^{17}$

In Europe, the positive results of the PROFILE 1001 (Phase I/II) and preliminary results of the PROFILE 1005 (Phase II), which were later congruent with the results from the PROFILE 1007 trial, lead to crizotinib's conditional approval in previously treated locally advanced and metastatic ALK-rearranged NSCLC. In the ongoing PROFILE 1005 study, crizotinib was evaluated for safety in 136 patients 
and for tumor response in 76 patients with advanced, recurrent and/or metastatic ALK-rearranged NSCLC previously treated with at least one prior chemotherapy regimen. The majority of patients were nonsmokers and mostly with adenocarcinoma histology. Tumor response was detected in $83 \%$ of tumors, half of which had tumor shrinkage $\geq 30 \%$. Most treatmentrelated toxicities were grade 1 or 2 , mainly nausea $(46 \%)$, vision disorders (45\%), vomiting (39\%), and diarrhea (29\%). In $15 \%$ of patients, grade 3 or 4 toxicities included elevated ALT, dyspnea, and neutropenia. Two deaths were attributed to treatment-related pneumonitis. Patient-reported outcomes showed clinically significant improvement in symptoms of pain, cough, dyspnea, and fatigue and overall preservation of quality of life. ${ }^{18}$

With the ground-breaking results of the PROFILE 1001, PROFILE 1005, and PROFILE 1007 trials at hand, it was only natural to evaluate the introduction of crizotinib as a treatment option for previously untreated tumors. The PROFILE 1014 trial was a Phase III trial comparing crizotinib against standard of care platinum doublet in previously untreated advanced ALK-rearranged non-squamous NSCLC. This trial demonstrated the superiority of crizotinib in prolonging PFS (10.9 months vs 7 months; HR 0.454, 95\% CI $0.346-0.596, P<0.0001)$ and an approximately $30 \%$ higher ORR. These results were announced at the 2014 American Society of Clinical Oncology meeting and established upfront crizotinib as the standard of care.

\section{Crizotinib and central nervous system disease}

Brain metastasis is a common complication of lung adenocarcinoma occurring in approximately $45 \%$ of patients. ${ }^{19}$ In a retrospective analysis of data from the PROFILE 1005 and PROFILE 1007 studies, a subset of 275 patients with asymptomatic brain metastases at baseline randomized to receive crizotinib had an intracranial disease control rate of approximately $60 \%$ but an intracranial ORR of $7 \%$ while achieving systemic response rates of approximately $50 \%{ }^{20}$ In 146 crizotinib-treated patients who developed disease progression (PD) from the PROFILE 1001 and PROFILE 1005 trials, recurrences occurred most commonly in single organs, and the most common single organ progression occurred in the brain despite good systemic control with continuing treatment. ${ }^{21}$ The poor cerebrospinal fluid (CSF) penetration of crizotinib was documented in a case report of an 18-year-old male with EML4-ALK-rearranged metastatic NSCLC who had stable systemic disease while on crizotinib but developed central nervous system (CNS) progression.
He was treated with crizotinib $250 \mathrm{mg}$ twice daily for 2 weeks, following which CSF and serum concentrations of crizotinib were measured showing a CSF-to-serum concentration of 0.0026 .22

Results of the ongoing PROFILE 1014 trial pertaining to the intracranial efficacy of crizotinib were released at the 2014 European Society for Medical Oncology meeting (abstract 12250) showing a numerical improvement of the intracranial time to progression in patients with baseline intracranial events in the crizotinib-treated group (HR 0.60, 95\% CI 0.34-1.05, $P$-value nonsignificant) compared to the standard chemotherapy group (HR 0.45, 95\% CI 0.19-1.07, $P$-value nonsignificant). Thus, although the current data suggest that crizotinib can decrease the rates of CNS failure when compared with systemic chemotherapy, many patients end up progressing in the brain despite a good systemic control.

\section{Mechanisms of resistance}

Similar to the inevitable acquired resistance to erlotinib in EGFR-mutated NSCLC, the antitumor effect of crizotinib is relatively short lived due to acquired resistance, with most patients progressing within the first 11 months. ${ }^{17}$ Lack of tumor shrinkage in a subset of patients with EML4-ALK rearrangement in the ongoing PROFILE 1001 study indicates the potential existence of primary resistance to ALK inhibition as well. ${ }^{19}$ Proven mechanisms of acquired ALK inhibition resistance include target mutation, target amplification, and target bypassing, which may coexist. ${ }^{23,24}$

In a study published by Katayama et al four ALK tyrosine kinase domain gene mutations accounted for $22 \%$ of resistant cases, with each mutation conferring a variable degree of resistance to crizotinib. These mutations were found to cluster around the ATP-binding pocket of ALK; two of them (G1202R, S1206Y) resulted in decreased affinity of crizotinib to its binding site on the mutant ALK, another (L1196M) was a gatekeeper mutation similar to the EGFR and BCR-ABL gatekeeper mutations leading to resistance to its targeting agents, and the forth (1151Tins) was located further away from the crizotinib-binding site and is proposed to diminish the affinity of ALK to ATP. ${ }^{23}$ Additional mutations conferring resistance to crizotinib have been identified. ${ }^{24-27}$

Wild-type ALK gene amplification was detected in a cell line of crizotinib-resistant samples with otherwise intact downstream signaling explaining crizotinib resistance in that line. ${ }^{23}$ Copy number gain (CNG) was defined as an increase of more than twofold of the mean of rearranged genes per cell in a crizotinib posttreatment sample compared to pretreatment in the study by Doebele et al and was detected in 
$18 \%$ of patients, half in whom it was the only abnormality. In posttreatment samples with $\mathrm{CNG}$, more copies of ALK rearrangement per cell as well as more cells displaying the rearrangement were detected by the standard break-apart FISH probe. $^{24}$

Bypassing the ALK signaling pathway by amplification of wild-type EGFR and its ligands resulted in ALK-independent activation of downstream signaling cascades in cell lines otherwise sensitive to crizotinib, similar to the MET amplification process bypassing EGFR and conferring resistance to anti-EGFR therapy in EGFRmutated NSCLC. The combination of crizotinib and gefitinib resulted in significant growth suppression, suggesting EGFR amplification as a resistance mechanism. ${ }^{23}$ KIT and KRAS amplification are the other two mechanisms reported that can bypass ALK and maintain downstream signaling. ${ }^{23,24}$

\section{The role of ceritinib in ALK- mutated NSCLC}

Ceritinib (LDK378) is a second-generation, ATP-competitive, oral tyrosine kinase inhibitor with higher ALK selectivity 20 times more potent than crizotinib. ${ }^{28,29}$ Early results from the ASCEND-1 trial demonstrated the efficacy of ceritinib in this Phase I international, multicenter trial that treated both crizotinib-naïve and crizotinib-treated advanced ALK-rearranged NSCLC. Response rates of $62 \%$ in crizotinib-naïve and $56 \%$ in crizotinib-treated patients were seen, with an ORR of $58 \%$ in patients who received ceritinib in doses of $400 \mathrm{mg}$ or more. The trial led to the FDA approval of ceritinib on April 29, 2014 for patients with ALK-positive, metastatic NSCLC with PD or who are intolerant to crizotinib. ${ }^{30,31}$ In crizotinib-resistant tumor samples, ceritinib demonstrated efficacy in overcoming their resistance in the more common mutations (L1196M, G1269A, S1206Y, and I1171T), whereas tumors with other mutations were also resistant to ceritinib (C1156Y, G1202R, 1151Tins, L1152R, and F1174C). ${ }^{23,24}$ Gastrointestinal adverse events were experienced in the majority of patients; grade 1 or 2 nausea ( $82 \%$ ), diarrhea $(75 \%)$, and/or vomiting $(65 \%)$ and fatigue were reported in about half the patients. Grade 3 or 4 toxicities were mostly elevated ALT (21\%), elevated AST (11\%), diarrhea (7\%), and elevated lipase (7\%) resulting in discontinuation of treatment in $6 \%$ due to toxicities. ${ }^{30}$ Persistent gastrointestinal adverse events and elevated aminotransferases limited dosing beyond $750 \mathrm{mg}$ as the minimal tolerated dose. Four cases of interstitial lung disease were reported and one case of QT prolongation. All adverse events resolved with discontinuation of treatment and administration of standard treatments. Approximately half of the total patient population required dose reduction, and $62 \%$ of the patients on the $750 \mathrm{mg}$ cohort had their dose reduced, with a median duration of interruption of about 1 week. At doses above $400 \mathrm{mg}, 1 \%$ of patients had a CR, $57 \%$ had a PR, 22\% had stable disease, and 11\% had PD with a median duration of response in patients achieving a CR or PR of 8.2 months and a median PFS of 7.0 months. At doses less than $400 \mathrm{mg}, 25 \%$ of patients had a PR (two out of eight patients at $200 \mathrm{mg}$ and $300 \mathrm{mg}$ ). ${ }^{30}$ Responses were similar in crizotinib-naïve and crizotinib-treated patients. ${ }^{30}$ Results of an extension and a subgroup analysis to the ASCEND-1 trial were presented at the annual American Society of Clinical Oncology 2014 meeting. In the extension, 246 patients with advanced ALK-rearranged NSCLC were stratified into two groups, crizotinib-pretreated $(n=163)$ and crizotinib-naïve $(n=83)$. Patients were treated with ceritinib $750 \mathrm{mg}$ daily for at least 18 weeks. The median ORR was higher in the crizotinib-pretreated group compared to the crizotinib-naïve group (67\% vs 41\%). The median ORR for the entire cohort was $60 \%$, median duration of response was 9.7 months, median PFS was 7 months, and time to initial response was 6.1 weeks. Reported adverse events were similar to initial reports. ${ }^{32}$ A subgroup analysis of the ASCEND-1 trial set out to delineate interethnic variations in safety and efficacy between Asians $(n=82)$ and Caucasians $(n=156)$. Patient baseline characteristics were similar, but more Caucasians were crizotinib pretreated. Overall, Asians tolerated treatment better than Caucasians with lower grade 3-4 adverse events reported (55\% and 72\%, respectively), and ceritinib was more efficacious in the Asian subgroup compared to Caucasians with a median ORR of $69 \%$ and $57 \%$, respectively, and a median duration of response of 10.1 months and 6.9 months, respectively. The investigators determined that these differences were not attributed to crizotinib-pretreatment differences. ${ }^{33}$

Two Phase III trials are underway comparing ceritinib to standard chemotherapy in previously untreated and previously treated adult patients with ALK-rearranged, advanced-stage NSCLC (ASCEND-4, ASCEND-5).

\section{Future directions}

In an era of targeted therapies and personalization in cancer treatment, the ALK resistance is one platform model supporting this direction. The increasing application of genomic sequencing and advances in drug development resulted in at least 12 agents currently being investigated as targets for this relatively uncommon subtype of cancer in order to 
overcome the limitations of crizotinib. Ceritinib is the only FDA-approved treatment for crizotinib-resistant cases to date. Furthermore, the FDA recently granted breakthrough therapy designation to AP26113 for the treatment of crizotinibresistant, metastatic ALK-rearranged NSCLC based on early results of the ongoing ALTA trial (NCT02094573) which were reported in the 2014 European Cancer Congress showing a $72 \%$ systemic objective response and a $71 \%$ objective response in brain metastases. ${ }^{34}$

Alectinib, a more potent and selective oral ALK inhibitor, was recently approved in Japan and granted breakthrough therapy designation by the FDA based on Phase I/II data from the AF-001JP trial showing a 93.5\% ORR in crizotinibresistant patients. ${ }^{35}$ Crizotinib-resistant CNS recurrence disease was evaluated in mouse models and showed efficacy in brain tumor regression and survival consistent with results of three patients with CNS disease in the AF-001JP trial, attributed to alectinib's high potency and its evasion of CNS efflux mechanisms achieving higher concentrations in the CSF. ${ }^{35,36}$ Alectinib was also effective in crizotinib-resistant patients with the gatekeeper mutation (L1196M) as well as the C1156Y, F1174L, G1269A, and 1151Tins mutations. ${ }^{37,38}$

PF06463922 is another ALK inhibitor in development designed to be a low-efflux substrate for P-glycoprotein to achieve high CSF concentrations; it was also shown to be effective against all mutation-driven crizotinib-resistant tumors. ${ }^{39}$ Heat shock protein 90 (Hsp90) inhibitor monotherapy has been studied with results indicating inferior ORR and less tolerable AE profile as well as poor CNS activity. ${ }^{39}$ Trials evaluating combination therapy with ALK inhibitors postulating the synergistic effect of direct kinase inhibition and disruption of cell signaling are currently underway. ${ }^{40}$

The advent of novel immunomodulatory therapies and the positive responses seen in a multitude of cancers lead to trials evaluating their responses in NSCLC. Early data from ongoing Phase I trials using immune checkpoint inhibitors targeting the programmed cell death receptor and its ligand (PD-1 and PDL-1, respectively) as well as cytotoxic T-lymphocyte-associated antigen 4 (CTLA4) show promising responses. A Phase I trial of the anti-CTLA4 agent ipilimumab plus crizotinib is currently accruing patients.

\section{New challenges in drug development}

The large-scale paradigm shift in cancer treatment in the past decades hallmarked by strong collaboration between lab researchers, clinical investigators, and industry sponsors is producing numerous compounds with unique potencies, selectivity, and toxicity profiles. These new agents are being studied in well-designed clinical trials set out to achieve objective responses and are regulated by committees supporting these efforts and expediting the drugs' availability for cancer patients.

The breakthrough therapy designation and accelerated approval programs for drug development highlighted a uniquely rapid introduction of the first ALK inhibitor, crizotinib, followed by a second-generation inhibitor for a lethal malignancy that suffered a paucity of breakthroughs prior to the discovery of targeted therapies. These designations were granted based on objective responses and PFSs and without proven OS benefits. While ALK rearrangement is viewed by most as a poor prognostic indicator, we have clearly seen improved survival outcomes with the introduction of ALK inhibitors. The cross-over nature of the study designs limits our ability to detect significant OS outcomes, and long-term prospective surveillance is needed.

Further, what is the optimal timing to introduce targeted agents in relation to other treatment modalities in ALKrearranged NSCLC? Fast-forward a decade from now, it is predicted that a myriad of systemic therapies will be available at the dispense of clinicians, including selective ALK inhibitors, immunomodulatory agents, Hsp90 inhibitors, and possibly novel chemotherapeutic agents. Crizotinib provided patients with a median PFS of 11 months (PROFILE 1014), after which the inevitable resistance develops. To date, the only agent approved in crizotinib-resistant tumors is ceritinib, an ALK inhibitor which demonstrated activity in crizotinib-naïve tumors, raising the question of whether ceritinib should be advanced to the first-line setting (NCT01828099) or reserved as another line of treatment to overcome crizotinib-resistant tumors, assuming the applicability of the Response Evaluation Criteria in Solid Tumors (RECIST) criteria in evaluating responses with kinase inhibitors. Arguments for the latter approach are ceritinib's less tolerable AE profile, the sensitivity of crizotinib-resistant tumors to other ALK inhibitors in contrary to resistance patterns in EGFR-mutated tumors, as well as the lack of insight on modes of resistance to ceritinib as a significantly more potent inhibitor. Lastly, providing that Hsp90 inhibitors and immunotherapies demonstrate positive responses, should a combinatorial approach be implemented upfront to provide more durable responses or should the different modalities be utilized sequentially as different lines of treatment? As of now, mature data on Hsp90 inhibitors are not available, but early results favor a combination approach, if any, given their lower response rates and less tolerable side effect profile 


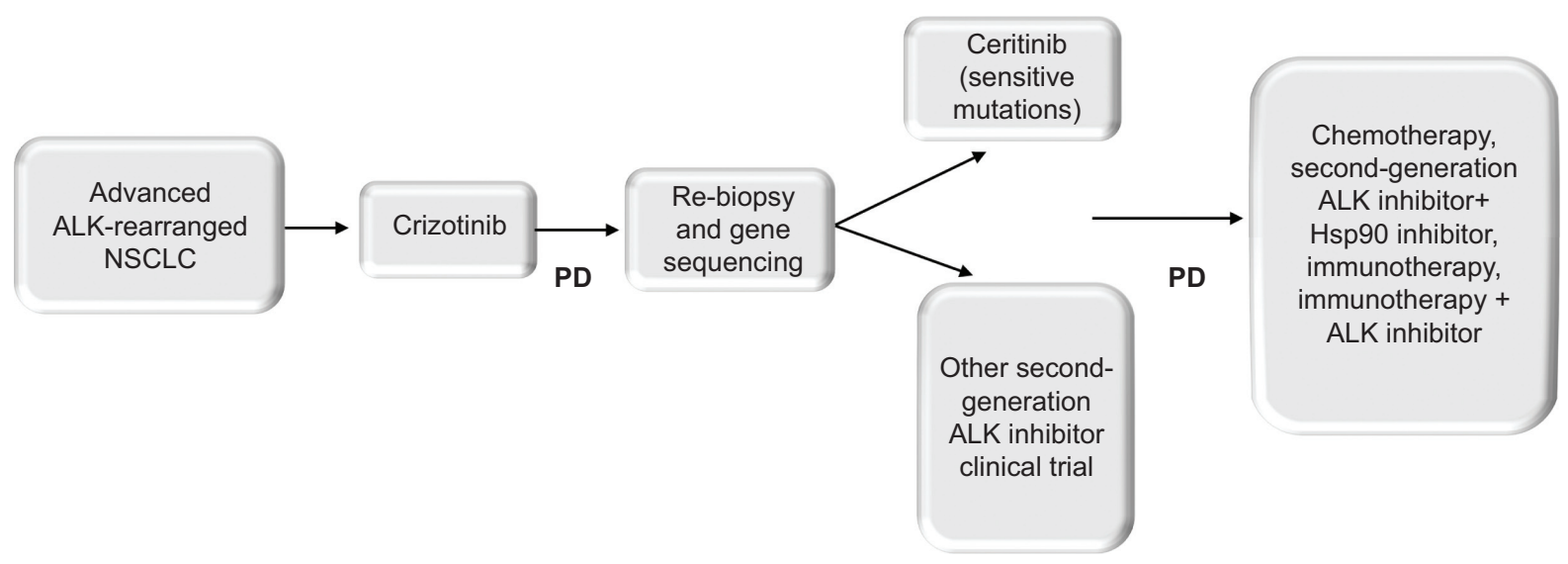

Figure I Proposed treatment algorithm for ALK-rearranged advanced NSCLC.

Abbreviations: ALK, anaplastic large-cell kinase; NSCLC, non-small-cell lung cancer; PD, disease progression; Hsp90, heat shock protein 90.

compared to ALK inhibitors. Ipilimumab, nivolumab, and pembrolizumab are immune checkpoint inhibitors gaining ground in NSCLC, although their activity in the specific subtype on ALK-rearranged NSCLC is unlikely to be different than non-ALK-rearranged tumors (Figure 1).

In addition, we are in the infancy stages of true personalized medicine in "oncogene-addicted" cancers. Despite the lag of its initial introduction in lung cancer, at the current pace of development, it may surpass the efforts in other fields to establish itself in a more advanced position. Most known mutations that confer resistance to crizotinib are actionable mutations using certain drugs while resistant to other drugs in the same class (Table 1). The development of ALK detection methods with higher sensitivity will likely lead to increasing application of ALK inhibitors, and the application

Table I Overcoming crizotinib resistance patterns with selective therapies in ALK-rearranged NSCLC

\begin{tabular}{ll}
\hline $\begin{array}{l}\text { Resistance } \\
\text { mechanism }\end{array}$ & Targeted treatment \\
\hline II5ITins & Not identified \\
CII56Y & Alectinib, X396 \\
FII74C & Alectinib, ASP3026; resistant \\
to ceritinib \\
FII74L & Not identified \\
GI269A & Ceritinib \\
GI202R & Resistant to ceritinib \\
III7IT & Ceritinib; resistant to alectinib \\
LII96M & Alectinib, AP26II3, ceritinib, \\
& ASP3026, X396 \\
LII52R & Not identified \\
SI206Y & Ceritinib \\
Bypassing EGFR & AP26II3 - dual ALK/EGFR inhibitor \\
pathway & \\
\hline
\end{tabular}

Abbreviations: NSCLC, non-small-cell lung cancer; ALK, anaplastic large-cell kinase; EGFR, epidermal growth factor receptor. of genomic sequencing in crizotinib-resistant cases can lead to "personalization" of the treatment approach.

Lastly, does targeted therapy go hand-in-hand with cost containment in medicine? Theoretically, the focus on cost-benefit and the resultant increase in development of supportive tools at the time of drug development (as a biomarker detection kit) would lead to a more confident and more cost-effective approach in delivering treatments with selective agents against actionable targets and therefore a decrease in overall cost. This was not translated in a Canadian study assessing the cost-effectiveness of EML4-ALK fusion gene testing along with first-line crizotinib using the Markov model. The study showed a gain of 0.011 qualityadjusted-life years (QALYs) compared with standard of care with molecular testing along with first-line crizotinib in patients with metastatic non-squamous NSCLC. The incremental cost was Canadian $\$ 2,725$ per patient and the incremental cost-effectiveness ratio (ICER) was $\$ 255,970$ per QALY gained. Patients with EML4-ALK advanced NSCLC tested and treated with crizotinib gained 0.379 additional QALYs and cost an additional \$95,043 compared with standard of care and produced an ICER of \$250,632 per QALY gained. The major driver of cost-effectiveness noted was drug price. ${ }^{41}$ A more selective strategy in testing patients for EML4-ALK is more economically feasible in a time of high health care costs.

In conclusion, the compilation of data has led to a general consensus among experts and a shift in the standard of care management of a newly diagnosed patient with ALK-rearranged NSCLC, becoming largely targeted therapy driven with upfront crizotinib and reserving ceritinib for resistant disease as of the time of submission of this manuscript. 


\section{Disclosure}

None of the authors report potential conflict of interests.

\section{References}

1. Mourali J, Bénard A, Lourenço FC, et al. Anaplastic lymphoma kinase is a dependence receptor whose proapoptotic functions are activated by caspase cleavage. Mol Cell Biol. 2006;26:6209-6222.

2. Mehlen P, Bredesen DE. Dependence receptors: from basic research to drug development. Sci Signal. 2011;4:mr2.

3. Soda M, Choi YL, Enomoto M, et al. Identification of the transforming EML4-ALK fusion gene in non-small-cell lung cancer. Nature. 2007;448:561-566.

4. Koivunen JP, Mermel C, Zejnullahu K, et al. EML4-ALK fusion gene and efficacy of an ALK kinase inhibitor in lung cancer. Clin Cancer Res. 2008; 14:4275-4283.

5. Inamura $\mathrm{K}$, Takeuchi $\mathrm{K}$, Togashi $\mathrm{Y}$, et al. EML4-ALK fusion is linked to histological characteristics in a subset of lung cancers. $J$ Thorac Oncol. 2008;3:13-17.

6. Wong DW, Leung EL, So KK, et al; University of Hong Kong Lung Cancer Study Group. The EML4-ALK fusion gene is involved in various histologic types of lung cancers from nonsmokers with wild-type EGFR and KRAS. Cancer. 2009;115:1723-1733.

7. Boland JM, Erdogan S, Vasmatzis G, et al. Anaplastic lymphoma kinase immunoreactivity correlates with ALK gene rearrangement and transcriptional upregulation in non-small cell lung carcinomas. Hum Pathol. 2009;40:1152-1158.

8. Rodig SJ, Mino-Kenudson M, Dacic S, et al. Unique clinicopathologic features characterize ALK-rearranged lung adenocarcinoma in the western population. Clin Cancer Res. 2009;15:5216-5223.

9. Takahashi T, Sonobe M, Kobayashi M, et al. Clinicopathologic features of nonsmall-cell lung cancer with EML4-ALK fusion gene. Ann Surg Oncol. 2010;17:889-897.

10. Inamura $\mathrm{K}$, Takeuchi $\mathrm{K}$, Togashi $\mathrm{Y}$, et al. EML4-ALK lung cancers are characterized by rare other mutations, a TTF-1 cell lineage, an acinar histology, and young onset. Modern Pathol. 2009;22(4):508-515.

11. Takeuchi K, Choi YL, Togashi Y, et al. KIF5B-ALK, a novel fusion oncokinase identified by an immunohistochemistry-based diagnostic system for ALK-positive lung cancer. Clin Cancer Res. 2009;15(9):3143-3149.

12. Rikova K, Guo A, Zeng Q, et al. Global survey of phosphotyrosine signaling identifies oncogenic kinases in lung cancer. Cell. 2007;131(6):1190-1203

13. Lindeman NI, Cagle PT, Beasley MB, et al. Molecular testing guideline for selection of lung cancer patients for EGFR and ALK tyrosine kinase inhibitors: guideline from the College of American Pathologists, International Association for the Study of Lung Cancer, and Association for Molecular Pathology. Arch Pathol Lab Med. 2013;137(6):828-860.

14. Demidova I, Barinov A, Savelov N, et al. Immunohistochemistry, fluorescence in situ hybridization, and reverse transcription-polymerase chain reaction for the detection of anaplastic lymphoma kinase gene rearrangements in patients with non-small cell lung cancer: potential advantages and methodologic pitfalls. Arch Pathol Lab Med. 2014; 138(6): 794-802.

15. Kwak EL, Bang YJ, Camidge DR, et al. Anaplastic lymphoma kinase inhibition in non-small-cell lung cancer. $N$ Engl J Med. 2010; 363(18):1693-1703.

16. Camidge DR, Bang Y, Kwak EL, et al. Efficacy and safety of crizotinib (PF-02341066) in patients with ALK-positive non-small cell lung cancer: updated results from the molecularly defined cohort of this first-in-man phase I study. Lancet Oncol. 2012;13:1011-1019.

17. Shaw AT, Kim DW, Nakagawa K, et al. Crizotinib versus chemotherapy in advanced ALK-positive lung cancer. $N$ Engl J Med. 2013 368(25):2385-2394.

18. Crinò L, Kim D, Riely GJ, et al. Initial phase II results with crizotinib in advanced ALK-positive non-small cell lung cancer (NSCLC): PROFILE 1005. J Clin Oncol. 2011;29(suppl):Abstr7514.
19. Sørensen JB, Hansen HH, Hansen M. Brain metastases in adenocarcinoma of the lung: frequency, risk groups, and prognosis. J Clin Oncol. 1988;6:1474-1480.

20. Costa DB, Shaw AT, Ou SH, et al. Clinical experience with crizotinib in patients with advanced ALK-rearranged non-small cell lung cancer and brain metastases in PROFILE 1005 and PROFILE 1007. J Thorac Oncol. 2013;8(suppl 2):S294-S295.

21. Otterson GA, Riely GJ, Shaw AT, et al. Clinical characteristics of ALK+ NSCLC patients (pts) treated with crizotinib beyond disease progression (PD): potential implications for management [ASCO abstract 7600] J Clin Oncol. 2012;30(15 suppl):12.

22. Costa DB, Kobayashi S, Pandya SS, et al. CSF concentration of the anaplastic lymphoma kinase inhibitor crizotinib. J Clin Oncol. 2011;29(15):e443-e445

23. Katayama R, Shaw AT, Khan TM, et al. Mechanisms of acquired crizotinib resistance in ALK-rearranged kung cancers. Sci Transl Med. 2012;4(120):120ra17.

24. Doebele RC, Pilling AB, Aisner DL, et al. Mechanisms of resistance to crizotinib in ALK gene rearranged non-small cell lung cancer. Clin Cancer Res. 2014;18:1472-1482.

25. Sasaki T, Koivunen J, Ogino A, et al. A novel ALK secondary mutation and EGFR signaling cause resistance to ALK kinase inhibitors. Cancer Res. 2011;71(18):6051-6060.

26. Choi YL, Soda M, Yamashita Y, et al; ALK Lung Cancer Study Group. EML4-ALK mutations in lung cancer that confer resistance to ALK inhibitors. N Engl J Med. 2010;363(18):1734-1739.

27. Kim S, Kim TM, Kim DW, et al. Heterogeneity of genetic changes associated with acquired crizotinib resistance in ALK-rearranged lung cancer. $J$ Thorac Oncol. 2013;8(4):415-422.

28. Marsilje TH, Pei W, Chen B, et al. Synthesis, structure-activity relationships, and in vivo efficacy of the novel potent and selective anaplastic lymphoma kinase (ALK) inhibitor 5-Chloro-N2(2-isopropoxy-5-methyl-4-(piperidin-4-yl)phenyl)-N4-(2-(isopropylsulf onyl)phenyl)pyrimidine-2,4-diamine (LDK378) currently in phase 1 and phase 2 clinical trials. $J$ Med Chem. 2013;56:5675-5690.

29. Friboulet L, Li N, Katayama R, et al. The ALK inhibitor ceritinib overcomes crizotinib resistance in non-small cell lung cancer. Cancer Discovery. 2013;4(12):1-12.

30. Shaw AT, Kim D-W, Mehra R, et al. Ceritinib in ALK-rearranged non-small-cell lung cancer. N Engl J Med. 2014;370(13):1189-1197.

31. US Food and Drug Administration. Ceritinib; 2014. Available from: http://www.fda.gov/drugs/informationondrugs/approveddrugs/ ucm395386.htm. Accessed July 1, 2014.

32. Kim DW, Mehra R, Tan DSW. Ceritinib in advanced anaplastic lymphoma kinase (ALK-rearranged (ALK+) non-small cell lung cancer (NSCLC): results of the ASCEND-1 trial. J Clin Oncol. 2014;32(15, suppl 1):8078

33. Tan DSW, Shaw AT, Mehra R. Ceritinib in Asian versus Caucasian patients with advanced anaplastic lymphoma kinase (ALK)-rearranged $(\mathrm{ALK}+)$ NSCLC: subgroup analysis of the ASCEND-1 trial. $J$ Clin Oncol. 2014;32(15, suppl 1):8078

34. Camidge DR, Bazhenova L, Salgia R, et al. Updated results of a firstin-human dose-finding study of the ALK/EGFR inhibitor AP26113 in patients with advanced malignancies. Paper presented at: European Cancer Congress; September 27-October 1; 2013; Amsterdam, the Netherlands: Abstract 3401

35. Seto T, Kiura K, Nishio M, et al. CH5424802 (RO5424802) for patients with ALK-rearranged advanced non-small-cell lung cancer (AF-001JP study): a single-arm, open-label, phase 1-2 study. Lancet Oncol. 2013; 14:590-598.

36. Kodama T, Hasegawa M, Takanashi K, Sakurai Y, Kondoh O, Sakamoto H. Antitumor activity of the selective ALK inhibitor alectinib in models of intracranial metastases. Cancer Chemother Pharmacol. 2014;74(5):1023-1028.

37. Sakamoto H, Tsukaguchi T, Hiroshima S, et al. CH5424802, a selective ALK inhibitor capable of blocking the resistant gatekeeper mutant. Cancer Cell. 2011;19:679-690. 
38. Kodama T, Tsukaguchi T, Yoshida M, Kondoh O, Sakamoto H. Selective ALK inhibitor alectinib with potent antitumor activity in models of crizotinib resistance. Cancer Lett. 2014;351:215-221.

39. Awad MM, Shaw AT. ALK Inhibitors in non-small cell lung cancer: crizotinib and beyond. Clin Adv Hematol Oncol. 2014;12(7):429-439.

40. Sang J, Acquaviva J, Friedland JC, et al. Targeted inhibition of the molecular chaperone Hsp90 overcomes ALK inhibitor resistance in non-small cell lung cancer. Cancer Discovery. 2013;3(4):430-443.
41. Djalalov S, Beca J, Hoch JS, et al. Cost effectiveness of EML4-ALK fusion testing and first-line crizotinib treatment for patients with advanced ALK-positive non-small-cell lung cancer. J Clin Oncol. 2014;32:1012-1019.

\section{Publish your work in this journal}

OncoTargets and Therapy is an international, peer-reviewed, open access journal focusing on the pathological basis of all cancers, potential targets for therapy and treatment protocols employed to improve the management of cancer patients. The journal also focuses on the impact of management programs and new therapeutic agents and protocols on

\section{Dovepress}

patient perspectives such as quality of life, adherence and satisfaction. The manuscript management system is completely online and includes a very quick and fair peer-review system, which is all easy to use. Visit http://www.dovepress.com/testimonials.php to read real quotes from published authors. 